\title{
Terahertz radar cross section measurements
}

\author{
Iwaszczuk, Krzysztof; Heiselberg, Henning; Jepsen, Peter Uhd
}

\section{Published in:}

Optics Express

Link to article, DOI:

10.1364/OE.18.026399

Publication date:

2010

Document Version

Publisher's PDF, also known as Version of record

Link back to DTU Orbit

Citation (APA):

Iwaszczuk, K., Heiselberg, H., \& Jepsen, P. U. (2010). Terahertz radar cross section measurements. Optics Express, 18(25), 26399-26408. https://doi.org/10.1364/OE.18.026399

\section{General rights}

Copyright and moral rights for the publications made accessible in the public portal are retained by the authors and/or other copyright owners and it is a condition of accessing publications that users recognise and abide by the legal requirements associated with these rights.

- Users may download and print one copy of any publication from the public portal for the purpose of private study or research.

- You may not further distribute the material or use it for any profit-making activity or commercial gain

- You may freely distribute the URL identifying the publication in the public portal

If you believe that this document breaches copyright please contact us providing details, and we will remove access to the work immediately and investigate your claim. 


\title{
Terahertz radar cross section measurements
}

\author{
Krzysztof Iwaszczuk, ${ }^{* 1}$ Henning Heiselberg ${ }^{2}$ and Peter Uhd Jepsen ${ }^{1}$ \\ ${ }^{1}$ DTU Fotonik - Department of Photonics Engineering, Technical University of Denmark, DK - 2800 Kongens \\ Lyngby, Denmark \\ ${ }^{2}$ Danish Defense Acquisition \& Logistics Organization, DK - 2750 Ballerup, Denmark \\ *kiwa@fotonik.dtu.dk
}

\begin{abstract}
We perform angle- and frequency-resolved radar cross section (RCS) measurements on objects at terahertz frequencies. Our RCS measurements are performed on a scale model aircraft of size $5-10 \mathrm{~cm}$ in polar and azimuthal configurations, and correspond closely to RCS measurements with conventional radar on full-size objects. The measurements are performed in a terahertz time-domain system with freely propagating terahertz pulses generated by tilted pulse front excitation of lithium niobate crystals and measured with sub-picosecond time resolution. The application of a time domain system provides ranging information and also allows for identification of scattering points such as weaponry attached to the aircraft. The shapes of the models and positions of reflecting parts are retrieved by the filtered back projection algorithm.
\end{abstract}

(C)2010 Optical Society of America

OCIS codes: (110.6795) Terahertz imaging; (110.6960) Tomography.

\section{References and links}

1. R. A. Cheville, and D. Grischkowsky, "Time domain terahertz impulse ranging studies," Appl. Phys. Lett. 67(14), 1960 (1995).

2. R. A. Cheville, R. W. McGowan, and D. R. Grischkowsky, "Late-time target response measured with terahertz impulse ranging," IEEE Trans. Antenn. Propag. 45(10), 1518-1524 (1997).

3. K. McClatchey, M. T. Reiten, and R. A. Cheville, "Time resolved synthetic aperture terahertz impulse imaging," Appl. Phys. Lett. 79(27), 4485 (2001).

4. T. M. Goyette, J. C. Dickinson, J. Waldman, and W. E. Nixon, “A $1.56 \mathrm{THz}$ compact radar range for W-band imagery of scale-model tactical targets," Proc. SPIE, Algorithms for Synthetic Aperture Radar Imagery VII, 4053, $615(2000)$

5. X. J. Zhong, T. J. Cui, Z. Li, Y. B. Tao, and H. Lin, "Terahertz-wave scattering by perfectly electrical conducting objects," J. Electromagn. Waves Appl. 21(15), 2331-2340 (2007).

6. J. Pearce, and D. M. Mittleman, "Scale model experimentation: using terahertz pulses to study light scattering," Phys. Med. Biol. 47(21), 3823-3830 (2002).

7. J. Pearce, and D. M. Mittleman, "Using terahertz pulses to study light scattering," Phys. B 338(1-4), 92-96 (2003).

8. A. G. Stepanov, J. Hebling, and J. Kuhl, "Efficient generation of subpicosecond terahertz radiation by phasematched optical rectification using ultrashort laser pulses with tilted pulse fronts," Appl. Phys. Lett. 83(15), 3000 (2003).

9. J. Hebling, A. G. Stepanov, G. Almasi, B. Bartal, and J. Kuhl, "Tunable THz pulse generation by optical rectification of ultrashort laser pulses with tilted pulse fronts," Appl. Phys. B 78, 593 (2004).

10. K.-L. Yeh, M. C. Hoffmann, J. Hebling, and K. A. Nelson, "Generation of $10 \mu \mathrm{J}$ ultrashort terahertz pulses by optical rectification," Appl. Phys. Lett. 90(17), 171121 (2007).

11. D. A. Bryan, R. Gerson, and H. E. Tomaschke, "Increased optical damage resistance in lithium niobate," Appl. Phys. Lett. 44(9), 847 (1984).

12. G. Gallot, and D. Grischkowsky, "Electro-optic detection of terahertz radiation," J. Opt. Soc. Am. B 16(8), 1204 (1999).

13. Q. Wu, and X.-C. Zhang, "Free-space electro-optic sampling of terahertz beams," Appl. Phys. Lett. 67(24), 3523 (1995).

14. P. U. Jepsen, C. Winnewisser, M. Schall, V. Schyja V, S. R. Keiding, and H. Helm, "Detection of THz pulses by phase retardation in lithium tantalate," Phys. Rev. E Stat. Phys. Plasmas Fluids Relat. Interdiscip. Topics 53(4), R3052-R3054 (1996).

15. Q. Wu, M. Litz, and X.-C. Zhang, "Broadband detection capability of ZnTe electro-optic field detectors," Appl. Phys. Lett. 68(21), 2924 (1996).

16. A. Nahata, D. H. Auston, T. F. Heinz, and C. Wu, "Coherent detection of freely propagating terahertz radiation by electro-optic sampling," Appl. Phys. Lett. 68(2), 150 (1996).

\#136711 - \$15.00 USD Received 18 Oct 2010; revised 15 Nov 2010; accepted 16 Nov 2010; published 1 Dec 2010

(C) 2010 OSA

6 December 2010 / Vol. 18, No. 25 / OPTICS EXPRESS 26399 
17. D. Turchinovich, and J. I. Dijkhuis, "Performance of combined [100]-[110] ZnTe crystals in an amplified THz time-domain spectrometer," Opt. Commun. 270(1), 96-99 (2007).

18. G. Zhao, M. Mors, T. Wenckebach, and P. C. M. Planken, "Terahertz dielectric properties of polystyrene foam," J. Opt. Soc. Am. B 19(6), 1476 (2002).

19. E. F. Knott, Radar cross section measurements (Van Nostrand Reinhold, New York, 1993).

20. D. L. Mensa, High resolution radar cross-section imaging (Artech House, Boston, 1991).

21. G. Mie, "Beiträge zur Optik trüber Medien, speziell kolloidaler Metallösungen," Ann. Phys. 25(3), 377-445 (1908).

22. M. Born, and E. Wolf, "Diffraction by a Conducting Sphere; Theory of Mie." $\$ 13.5$ in Principles of Optics: Electromagnetic Theory of Propagation, Interference, and Diffraction of Light, 7th ed. (Cambridge, England: Cambridge University Press, 1999) p. 633-644.

23. R. Bracewell, The Hilbert Transform The Fourier Transform and its Applications, 3rd ed. (New York, McGrawHill, pp. 267-272, 1999).

24. J. Pearce, H. Choi, D. M. Mittleman, J. White, and D. Zimdars, "Terahertz wide aperture reflection tomography," Opt. Lett. 30(13), 1653-1655 (2005).

25. A. C. Kak, and M. Slaney, "Principles of Computerized Tomographic Imaging", IEEE Press (1988).

\section{Introduction}

Scattering of terahertz $(\mathrm{THz})$ waves by conducting and dielectric objects is an area of intensive investigation both from theoretical and experimental side [1-7]. Time-domain based $\mathrm{THz}$ systems can provide a convenient, fast and precise method of visualization of scattering centers. At the same time, the measurement of the radar cross section (RCS) is a standard technique, particularly important for military and defense-related purposes, including detection and identification of aircrafts, ships and other targets as well as for countermeasures such as $R C S$ reduction and stealth. The necessity of operating on massive objects, such as full-size airplanes or ships, can make those measurements complicated, time consuming, and expensive. Also the size of the platforms makes it impractical to iterate the design and testing phases, allowing only for software calculations of $R C S$ values with limited possibility of experimental testing.

In this paper we combine time-domain based $\mathrm{THz}$ system and RCS technique to obtain $R C S$ values at $\mathrm{THz}$ frequencies. By employing scaling laws we are able to scale values measured in the low $\mathrm{THz}$ range $(0.1-2 \mathrm{THz})$ into values that would be measured at radar frequencies. Typical radar systems operate at frequency range from hundreds of $\mathrm{MHz}$ up to a few tens of GHz. This defines the scaling factor between $\mathrm{THz}$ waves and radar waves from tens to few hundreds, forcing scale objects to be in size range of few centimeters and larger. Object of such dimensions is easy to handle and manipulate, and allows for iterative design and testing procedure, where the test object is manufactured by a rapid prototyping system such as a computer numerically controlled $3 \mathrm{D}$ milling machine or a $3 \mathrm{D}$ printer.

In this work we measure THz RCS of scale models and present polar and azimuthal timeand frequency resolved $R C S$ plots of F-16 fighter aircraft model rotated on a pedestal. The time domain sub-ps resolution allows for sub-mm range resolution that can be found in modern software defined radars and also allows for identification of scattering points. The shape of the model and positions of scattering parts are retrieved by the filtered back projection algorithm.

\section{Experimental details}

A schematic diagram of our $\mathrm{THz}$ RCS setup is shown in Fig. 1. A regenerative Ti:sapphire femtosecond laser amplifier delivers $\sim 35 \mathrm{fs}, 2.9 \mathrm{~mJ}$ pulses with center wavelength of $800 \mathrm{~nm}$ at a $1 \mathrm{kHz}$ repetition rate. The laser output is split into a source beam for $\mathrm{THz}$ pulse generation and a gating beam for $\mathrm{THz}$ detection by free-space electro-optic sampling. $\mathrm{THz}$ waves are generated by optical rectification in lithium niobate LiNbO3 crystal applying the tilted pulse front method [8-10]. The intensity front of fs pulses is tilted by a 1714 line $/ \mathrm{mm}$ grating and imaged by a $75 \mathrm{~mm}$ lens with demagnification factor of 2 onto the front surface of a stoichiometric $\mathrm{LiNbO} 3$ crystal at room temperature. The $\mathrm{LiNbO} 3$ crystal was doped with $1 \%$ of $\mathrm{MgO}$ to prevent photorefractive damage [11]. The highest $\mathrm{THz}$ peak electric field in the focused THz beam achieved in our setup is at least $40 \mathrm{kV} / \mathrm{cm}$, limited predominantly by the high angular and material dispersion inside the LiNbO3 crystal of the $60 \mathrm{~nm}-\mathrm{FWHM}$ 
pump pulses. The high efficiency of $\mathrm{THz}$ generation allows us to obtain high dynamic range of the setup up to $43 \mathrm{~dB}$, defined as ratio between the peak electric field to the noise floor for the $\mathrm{THz}$ beam reflected in a specular angle off a metal mirror bigger than the $\mathrm{THz}$ beam. The near-infrared light transmitted though the LiNbO3 crystal is blocked by a black polyethylene sheet, highly transmissive for the $\mathrm{THz}$ pulse.

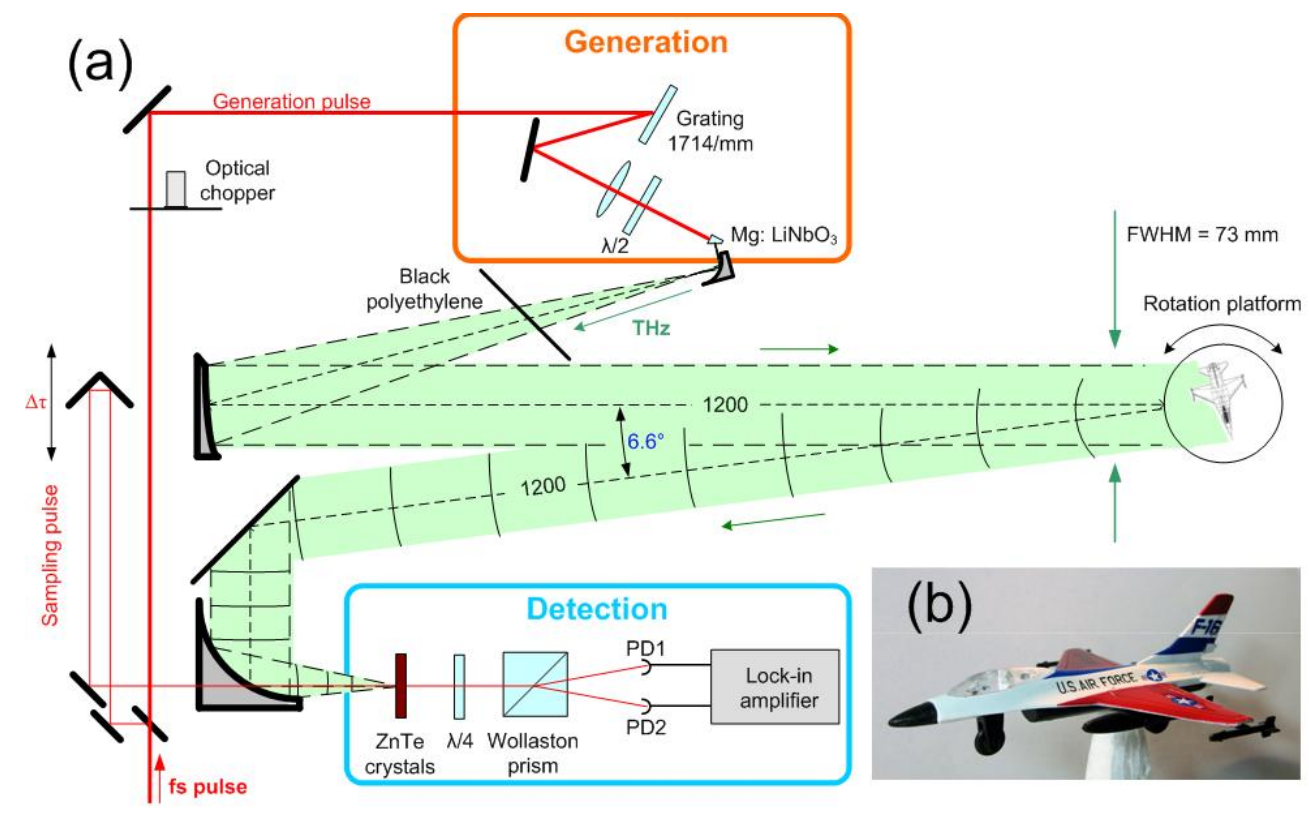

Fig. 1. (a) Schematic diagram of the THz RCS setup. (b) $10 \mathrm{~cm}$-long 1:150-scale metal model of aircraft fighter F-16.

A pair of off-axis parabolic mirrors with focal lengths of $25.4 \mathrm{~mm}$ and $516.8 \mathrm{~mm}$ and diameters of $25.4 \mathrm{~mm}$ and $101.6 \mathrm{~mm}$ respectively is used to expand the $\mathrm{THz}$ beam and subsequently to collimate it. The wide, collimated $\mathrm{THz}$ beam propagates $1200 \mathrm{~mm}$ towards the target which is placed on a computer-controlled rotation platform. The expansion optics increases the $\mathrm{THz}$ beam size by a factor of 20, which leads to a spatial FWHM of $73 \mathrm{~mm}$ of the THz electric field at the object distance. The electric field of the $\mathrm{THz}$ transient is polarized in the vertical plane. The scattered $\mathrm{THz}$ radiation is collected by a $170 \mathrm{~mm}$-diameter flat metal mirror at a distance of $1200 \mathrm{~mm}$ from the target, and focused using a $101.6 \mathrm{~mm}$ diameter, $152.4 \mathrm{~mm}$-focal length off-axis paraboloidal mirror onto a detection crystal. The scattered $\mathrm{THz}$ radiation thus travels $1430 \mathrm{~mm}$ before it reaches the detector. The angle between the incident and scattered radiation is $6.6^{\circ}$ and therefore our RCS measurements are in principle bistatic, but in practice reasonably close to a monostatic radar configuration. A truly monostatic configuration can be achieved by using a thick wedge silicon beam splitter and a normal incidence. Such a configuration will inevitably reduce the dynamic range due to reflection losses on the beam splitter. All $\mathrm{THz}$ and infrared beams propagate at a height of $160 \mathrm{~mm}$ above optical table which is sufficient to avoid reflections from the table surface. The setup is aligned using a big $170 \mathrm{~mm}$-diameter metal mirror in a place of the scattering object. This mirror reflects all $\mathrm{THz}$ radiation in the direction of the receiver. The complete $\mathrm{THz}$ beam path is purged by dry nitrogen to avoid absorption by water vapor in the atmosphere. 

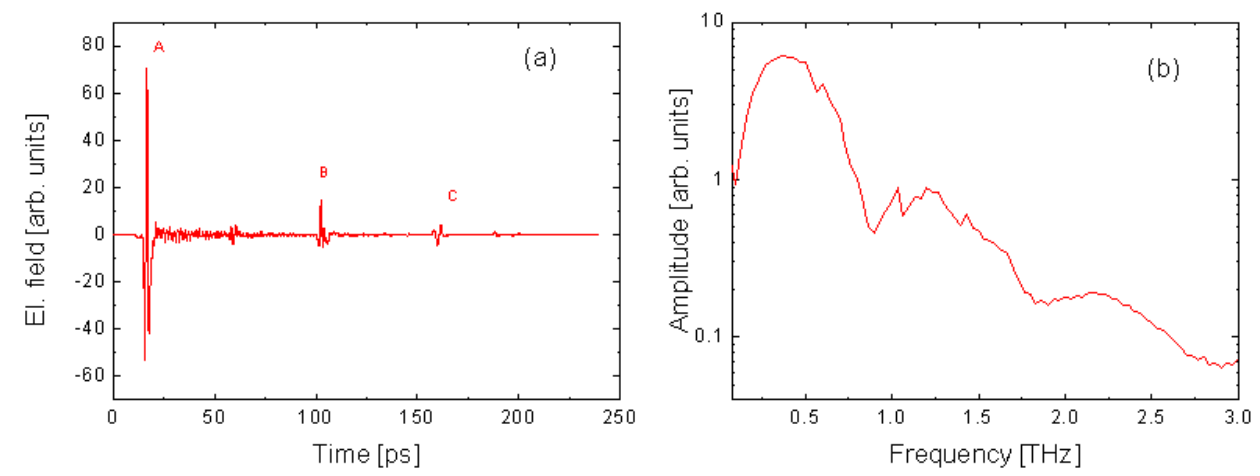

Fig. 2. (a) $\mathrm{THz}$ waveform reflected from a $170 \mathrm{~mm}$-diameter metal flat disk. The transient include the main $\mathrm{THz}$ pulse (labeled $\mathrm{A}$ ), a partial reflection of the main pulse inside the detection crystals (labeled B), and a part of the main pulse undergoing multiple reflections in the $\mathrm{LiNbO}_{3}$ crystal (labeled $\mathrm{C}$ ). (b) The amplitude spectrum of the generated $\mathrm{THz}$ radiation obtained as a Fourier transform of a 50 ps-wide time window around transient A.

The $800 \mathrm{~nm}$ probe beam is directed through a small hole in the center of off-axis parabolic mirror for collinear detection. A computer-controlled delay stage is used to map the temporal $\mathrm{THz}$ pulse shape. The electric field of the $\mathrm{THz}$ transients is detected by free-space electrooptic sampling [12-16] in a [110] zinc telluride (ZnTe) crystal (2 mm-thick). A second passive [100] 2mm-thick ZnTe crystal with anti-reflective coating for $800 \mathrm{~nm}$ light on one facet is stacked together with the active crystal to increase temporal separation between the directly transmitted THz pulse (transient A in Fig. 2(a)) and the pulse that experience two reflections in the detection crystals (transient B in Fig. 2(a)) [17]. The time delay between these two pulses is $89 \mathrm{ps}$. The temporal order of incoming pulses is further complicated by an additional THz pulse (transient $\mathrm{C}$ in Fig. 2(a)) which experiences multiple reflections inside $\mathrm{LiNbO}_{3}$ crystal and arrives at detector 160ps after the main pulse. Between transient A and pulse B (at $60 \mathrm{ps}$ ) is also a small pulse visible, which has its origins in imperfections of the boundary between the [110] detection crystal and the [100] inactive spacer crystal. The FWHM of the main $\mathrm{THz}$ transient of 0.96 ps allows for distinction of two different point scatterers separated by $144 \mu \mathrm{m}$ along the THz propagation direction. Figure 2(b) shows the amplitude spectrum of the detected $\mathrm{THz}$ radiation obtained from a Fourier transform of a 50 ps time window around transient A. The amplitude spectrum extends from $0.02 \mathrm{THz}$ up to 3 $\mathrm{THz}$, but $95 \%$ of the generated $\mathrm{THz}$ energy lies within $0.1 \mathrm{THz}-1.0 \mathrm{THz}$ range. The longest THz waves that are generated in our setup have wave vector of $k=2 \pi / \lambda=\sim 2 \mathrm{~mm}^{-1}$ (at $\sim 0.1$ $\mathrm{THz})$, so for all the investigated objects $(L>1 \mathrm{~mm})$ we are in the optical regime $(k L>>1)$.

The target objects are placed on a support column of polystyrene foam, which consists of $97 \%$ air or other gases and only 3\% polymer, and thus has low refractive index of 1.02 in the $\mathrm{THz}$ range [18]. Furthermore, the polystyrene foam is cut in a shape which resembles a tapered diamond column, which reflects all the $\mathrm{THz}$ radiation away from the detector. No signs of volume reflections from the thousands of internal cells in the foam structure have been observed. No $\mathrm{THz}$ signal has been measured without samples positioned on the polystyrene support column. The investigated target was a 1:150-scale model of aircraft fighter F-16. The model was $10 \mathrm{~cm}$ long with a wingspan of $6.7 \mathrm{~cm}$. The object was made of a painted metal with elements such as missiles, nose and undercarriage made of plastic. 


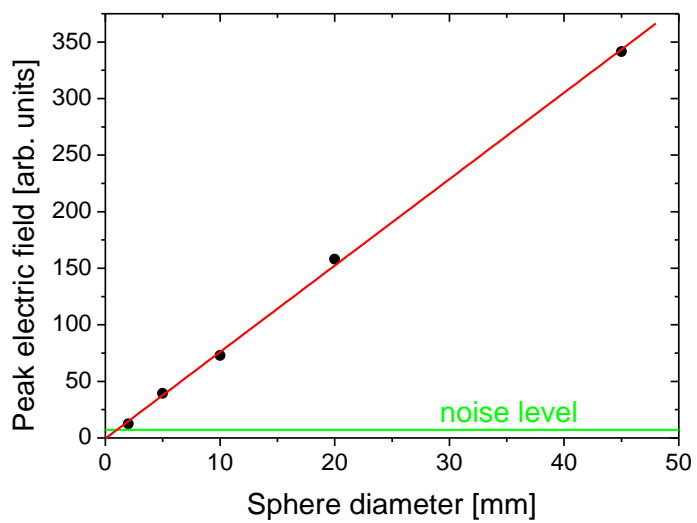

Fig. 3. The peak electric field of $\mathrm{THz}$ radiation scattered from a conducting sphere as a function of sphere diameter. The red line shows the best linear fit to the experimental data. The horizontal green line represents the average noise level in single scan measurement. The red and green curves intercept at the point corresponding to a sphere of $0.98 \mathrm{~mm}$ diameter.

\section{Results and discussion}

The RCS of a scattering target illuminated with radar radiation is a parameter expressed in units of area which describes the intensity of the wave reflected back to the radar. The most general definition of $R C S$ can be written as [19,20],

$$
R C S=\lim _{R \rightarrow \infty} 4 \pi R^{2} \frac{\left|E_{s}\right|^{2}}{\left|E_{i}\right|^{2}},
$$

where $E_{i}$ and $E_{s}$ are the incident and scattered electric fields, and $R$ is the distance between radar and target. In this definition the limiting process is introduced first of all to ensure that the incident wave is a plane wave, which in the optical design of our setup is fulfilled. The $R C S$ depends mostly on the size and shape of the target, incident and reflected angle, ratio between the size of target and the wavelength, polarization of the emitted and detected radiation and finally on the properties of the material that the target is made of. The simplest scatterer in terms of its $R C S$ is a perfectly conducting sphere. Scattering of electromagnetic waves on a metal sphere is well described by Mie theory [21,22], which states that for $k \cdot r \gg 1$ a metal sphere has a frequency independent $R C S$ equal to its physical cross section area, $R C S=\pi r^{2}$, where $r$ is sphere radius. For a conducting cylinder the largest $R C S=2 \pi r h^{2} / \lambda$ is achieved at the specular angle, where $h$ is the height of the cylinder. The $R C S$ of a flat conducting plate with sides a and $\mathrm{b}$ decreases rapidly with angle from the maximum value $R C S=4 \pi a^{2} b^{2} / \lambda^{2}$ at the specular reflection angle. The RCS should ideally be a parameter describing the target and should not depend on the target-detector distance. This statement is valid only for sufficiently large distances. Let us consider a flat disc with radius $r_{0}$, which for short distances can be treated as a perfect reflector. At the specular reflection angle, $\left|E_{s}\right|^{2}=\left|E_{i}\right|^{2}$, the RCS defined by Eq. (1) will increase quadratically with distance $R$. For longer distances wave diffraction becomes dominant and $\left|E_{s}\right|^{2} \propto 1 / R^{2}$ implying that the RCS saturates. Based on Fresnel diffraction theory it can be shown that the $R C S$ of a circular flat disc reaches $91 \%$ of its long-distance value at a distance $R=3 r_{0}^{2} / \lambda$. The distance where the $R C S$ reaches its far-distance value is shorter for objects with smaller

\#136711 - \$15.00 USD Received 18 Oct 2010; revised 15 Nov 2010; accepted 16 Nov 2010; published 1 Dec 2010 (C) 2010 OSA 6 December 2010 / Vol. 18, No. 25 / OPTICS EXPRESS 26403 
radius of curvature. Also the angular distribution of scattered electric field depends on the distance from the target.

Figure 3 shows the peak scattered electric field as a function of metal sphere diameter between 2 and $45 \mathrm{~mm}$. The steel spheres are placed on the polystyrene column and centered in the THz beam. The data are results of averaging up to 25 single $\mathrm{THz}$ waveforms. Since all the frequency components arrive in phase at the peak of the $\mathrm{THz}$ transient, the squared value of the peak electric field $E_{p}$ accurately represents the energy carried by the pulse. Since $R C S_{\text {sphere }}=\pi r^{2}$ and $R C S_{\text {sphere }} \sim E_{p}^{2}$, we find that $E_{p} \sim r$. This linear relationship is shown in Fig. 3 from which the coefficient of proportionality is obtained by linear regression (red line). For metal spheres with diameter larger than the $\mathrm{THz}$ beam waist dependence of $E_{p}$ of sphere radius will become sublinear, and will saturate at a constant value at the limit of $r \rightarrow \infty$, when all the $\mathrm{THz}$ radiation is reflected to the detector. The horizontal green line in Fig. 3 represents the noise level of a single scan. The smallest detectable sphere in a single scan has a diameter of $0.98 \mathrm{~mm}$ (crossing of the red and green line).

(a)

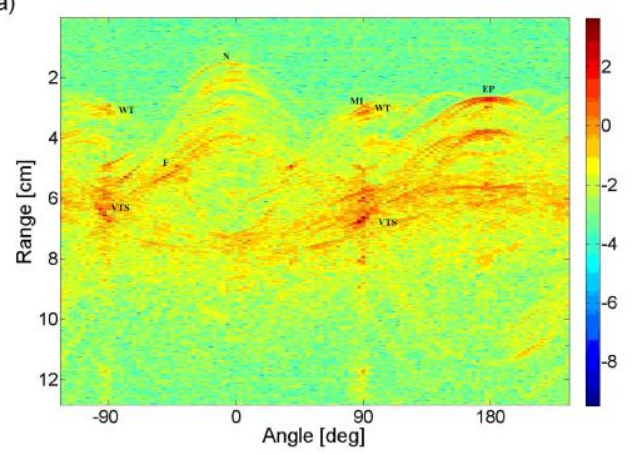

(c)

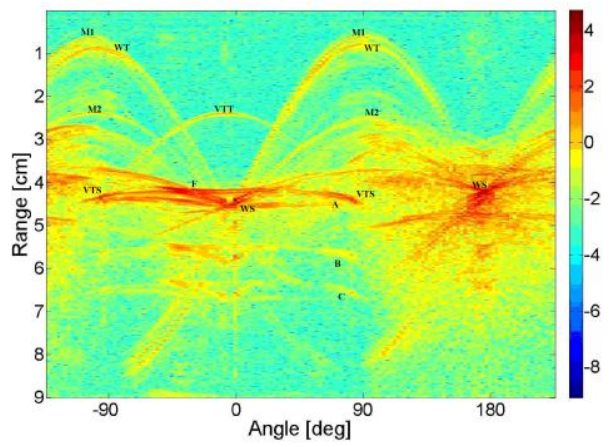

(b)

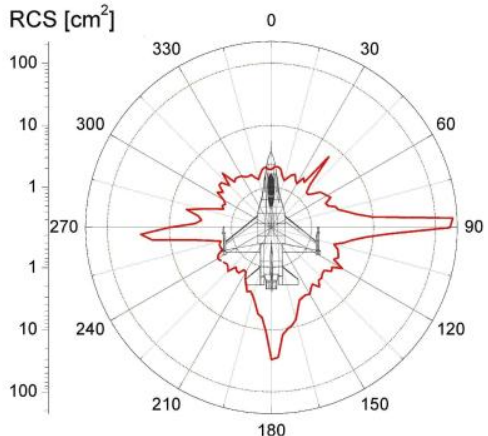

(d)

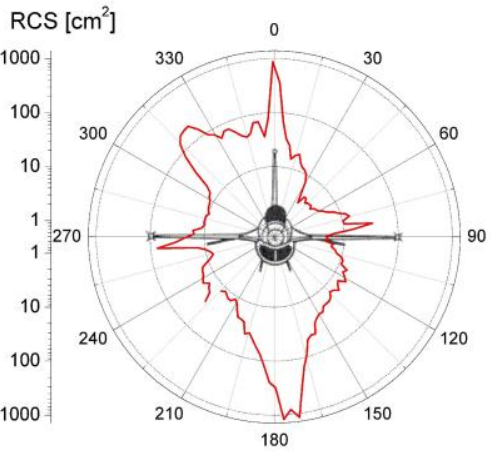

Fig. 4. Logarithm of the instantaneous amplitude of $\mathrm{THz}$ waveforms scattered from the F-16 scale model shown in Fig. 1(b) for different (a) polar and (c) azimuthal angles and (b), (d) their frequency-averaged $R C S$. Letter marks indicate positions of different scattering parts of the airplane model: nose (N), wing tips (WT), wing surface (WS), vertical tail surface (VTS), vertical tail tip (VTT), fuselage (F), missiles (M1, M2). Additionally letters marks A, B and C show example of the main transient and two echoes (according to Fig. 2 (a)).

For complicated structures, which we will be dealing below, the electric field from multiple point scatterers overlap and another method is required for calculating the RCS. For the purposes of this article we introduce a frequency-averaged RCS, which can be defined by: 


$$
R C S=\pi r_{0}^{2} \cdot \frac{\int_{0}^{T}\left|E_{\text {object }}(t)\right|^{2} d t}{\int_{0}^{T}\left|E_{c a l}(t)\right|^{2} d t-\int_{0}^{T}\left|E_{b g}(t)\right|^{2} d t},
$$

where $E_{\text {object }}(t)$ is the detected electric from the scattering object as a function of time $t$, $E_{c a l}(t)$ the electric field scattered by calibrated metallic spheres of RCS $\pi r_{0}{ }^{2}$, and $E_{b g}(t)$ represents background noise measured as a signal without the sample on the support column. The standard procedure to calibrate a RCS setup is substitution by a metal sphere of known $R C S$. Since we measure not only the intensity of electromagnetic radiation but the field itself we can also introduce the frequency resolved $R C S$ defined as follows:

$$
R C S(\omega)=\pi r_{0}^{2} \cdot \frac{\left|E_{\text {object }}(\omega)\right|^{2}}{\left|E_{c a l}(\omega)\right|^{2}-\left|E_{b g}(\omega)\right|^{2}},
$$

where $E_{i}(\omega)$ is the Fourier transform of $E_{i}(t)$.

Figure 4(a) shows the logarithm of the instantaneous amplitude of the $\mathrm{THz}$ waveforms vs. polar angle and range (calculated from the time-of-flight of the reflected $\mathrm{THz}$ pulse and the speed of light), recorded on the F-16 scale model. The instantaneous amplitude of the function $u(t)$ is the absolute value of the complex analytic signal $u_{a}(t)=u(t)+i \cdot H\{u\}(t)$, where $H\{u\}(t)$ is the Hilbert transform [23] of a function $u(t)$, and which can be expressed using following formula:

$$
H\{u\}(t)=\frac{1}{\pi} \text { p.v. } \int_{-\infty}^{\infty} \frac{u(\tau)}{t-\tau} d \tau,
$$

where p.v. stands for principal value. For example for a pure sinusoid, the instantaneous amplitude is a constant, while for single-cycle electromagnetic pulses the instantaneous amplitude is a good representation of the envelope function. In such high range resolution maps the single point scatterers are seen as sine functions (sinograms) of the rotation angle. $0^{\circ}$ position on Fig. 4(a) corresponds to the position where the nose of the airplane faces the incoming $\mathrm{THz}$ radiation, while $180^{\circ}$ is tail-on Clear traces of scattering originating at the airplane nose, wing tips, fuselage, tail and exhaust pipe are visible. Figure 4(b) shows the frequency-averaged polar $R C S$ for the investigated scale model. The values of $R C S$ vary from $1.4 \mathrm{~cm}^{2}$ (seen from the front of the airplane) up to $193 \mathrm{~cm}^{2}$ (seen from a wing side). The $R C S$ plot is rotated slightly counter-clockwise with respect to the orientation of the symmetry plane because of the $6.6^{\circ}$ bistatic design of the setup. The RCS plot is also asymmetric due to a possible minor unintentional azimuthal tilt of the scale model. Figure 4(c) presents again the logarithm of the instantaneous amplitude of the $\mathrm{THz}$ waveforms, now as function of the azimuthal angle and range. On the azimuthal map, the orientation $0^{\circ}$ corresponds to the situation at which the airplane is exposed to $\mathrm{THz}$ radiation directly from above. Scattering from wing surfaces and edges, tail, fuselage and even plastic missiles is easily distinguishable. However, due to pulse reflections B and C in Fig. 2(a) some of the sinograms are repeated with a delay corresponding to the delay between the reflections, but at significantly lower amplitudes. By inspection of Fig. 4(d) we notice that the largest $R C S$ in the azimuthal configuration is when the airplane is seen exactly from below $\left(1208 \mathrm{~cm}^{2}\right)$ and above $\left(881 \mathrm{~cm}^{2}\right)$. The smallest $R C S$ is observed just below the wings. The RCS plot is again counter-clockwise rotated with respect to the orientation of the symmetry plane due to the bistatic nature of measurement. Scaling by a factor of $150^{2}$ can provide the $R C S$ of a full-sized F-16 measured at a distance of $150 \cdot 143 \mathrm{~cm}=214.5 \mathrm{~m}$ at frequency $\sim 3 \mathrm{GHz}$. This scaling

\#136711 - \$15.00 USD Received 18 Oct 2010; revised 15 Nov 2010; accepted 16 Nov 2010; published 1 Dec 2010 (C) 2010 OSA 6 December 2010 / Vol. 18, No. 25 / OPTICS EXPRESS 26405 
approach is only valid as far as the model is a true scale copy of the original and under the assumption that the material reflection properties are unchanged as function of frequency. This scaling assumption is true for metals between the $\mathrm{GHz}$ and $\mathrm{THz}$ frequencies, but may be validated for the dielectric objects. Both data sets were measured in the configuration where the incoming $\mathrm{THz}$ radiation was polarized in the vertical direction. Obtained results would be different if the $\mathrm{THz}$ radiation was polarized in the horizontal direction. We can expect that the biggest differences would come from objects with dimensions in one direction longer than the $\mathrm{THz}$ wavelength and in the perpendicular direction comparable or shorter than the $\mathrm{THz}$ wavelength, such as thin wires or sharp edges (for example wing or tail tips). The FWHM of the instantaneous amplitude of the main THz transient from the Fig. 2(a) is 1.8 ps what gives range resolution of presented sinograms of $270 \mu \mathrm{m}$.

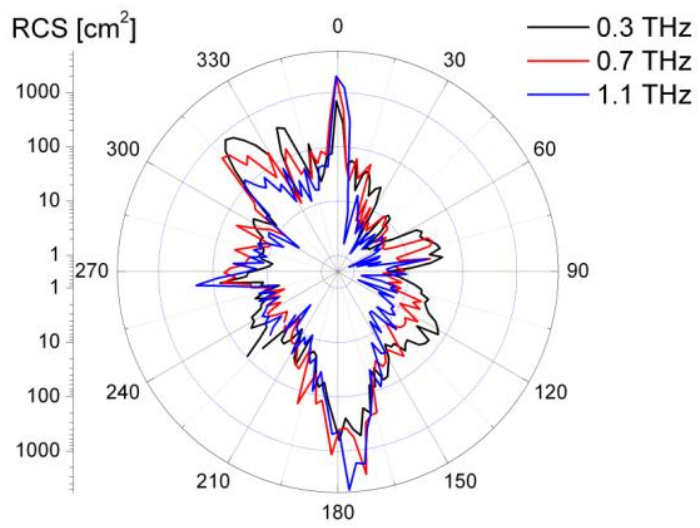

Fig. 5. Frequency-resolved azimuthal $R C S$ of a metal model of the fighter aircraft F-16 at frequencies $0.3,0.7$ and $1.1 \mathrm{THz}$. The presented data are averaged within a frequency interval of $+/-20 \mathrm{GHz}$.

Figure 5 shows values of the frequency-resolved THz RCS of the scale model of the F-16 fighter in the azimuthal configuration for frequencies $0.3,0.7$ and $1.1 \mathrm{THz}$. We notice that at angles where the $\mathrm{THz}$ waves are reflected from large plane surfaces $\left(0^{\circ}\right.$ and $180^{\circ}$ - from the wing surfaces and at $90^{\circ}$ and $270^{\circ}$ from the tail surface), the $R C S$ at high frequencies is larger than at low frequencies, in agreement with the frequency-dependent nature of wave scattering by objects with different curvatures.

Our $\mathrm{THz}$ radar system is similar to a reflection tomography setup [24]. Each sinogram contains enough information about the target to transform it into a two-dimensional image of the target. The filtered back projection algorithm (FBP) [25] is one possible method to retrieve the spatial distribution of scatterers. However FBP algorithm does not include wave diffraction effects what leads to artifacts that we demonstrate by measurements on a metal test target shown in Fig. 6(a). The test target is an elongated cuboid (20 x $20 \mathrm{~mm}$ in cross section) with two 4 x $4 \mathrm{~mm}$ grooves cut in its cross-section profile. Additionally on one of the sides a $4 \mathrm{~mm}$-thick step was introduced to make the structure more asymmetric. Figure 6(b) shows sinogram for the test target. Data are taken with $1^{\circ}$ angular resolution. Figure $6(\mathrm{c})$ shows the FBP reconstruction using data from Fig. 6(b). Because of the small sizes of the test object (for most of the angles echoes $\mathrm{B}$ and $\mathrm{C}$ do not overlap in time with the real reflected signals) it was possible to apply simple time windowing function that would minimize effects of echoes $\mathrm{B}$ and $\mathrm{C}$. The reconstructed image represents the actual shape of the target well. However, the image is not free from artifacts in the form of lines crossing the whole image, with the tendency for large plane surfaces to be represented by stronger and longer lines. This behavior originates in the fact that the FBP algorithm does not compensate for diffraction of scattered waves. That effect is especially important at intermediate target-detector distances, where the angular distribution of electric field depends strongly on object size and shape. In our case the detector is placed $143 \mathrm{~cm}$ away from the target. At this distance, the $R C S$ reaches

\#136711 - \$15.00 USD Received 18 Oct 2010; revised 15 Nov 2010; accepted 16 Nov 2010; published 1 Dec 2010

(C) 2010 OSA

6 December 2010 / Vol. 18, No. 25 / OPTICS EXPRESS 26406 
$91 \%$ of its long-distance value for a plane metal plate of radius $r_{0}=\sqrt{R \lambda / 3}=12 \mathrm{~mm}$ at a frequency of $1 \mathrm{THz}$. This implies that for flat objects bigger than $12 \mathrm{~mm}$, the angular distribution of the scattered electric field is strongly dependent on the size of the target even within a class of objects with the same shape. A more complete reconstruction algorithm would have to take such diffraction effects into account. To further illustrate this weakness of the FBP algorithm in the form that we have applied here, we window out the intense, localized reflections from flat surfaces in the sinogram in Fig. 6(b), since these reflections are the main source of error. Figure 6(d) shows the result of the FBP algorithm after windowing. The picture is much clearer and in a better way represents all the point and edge scatterers. The depth resolution of a single angle scan is $270 \mu \mathrm{m}$, but because FBP uses many projections for image reconstruction, both depth and lateral resolution of the reconstructed image is $270 / \sqrt{2}=191 \mu \mathrm{m}$.

(a)

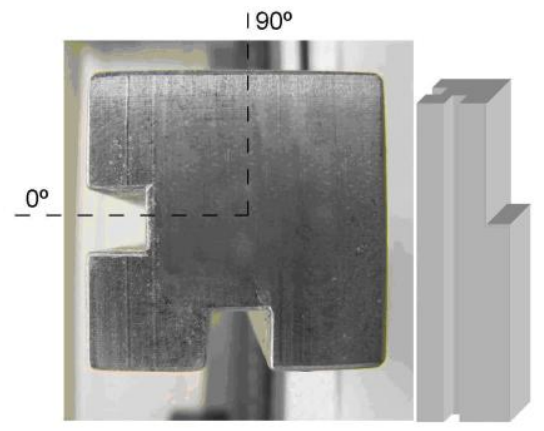

(c)

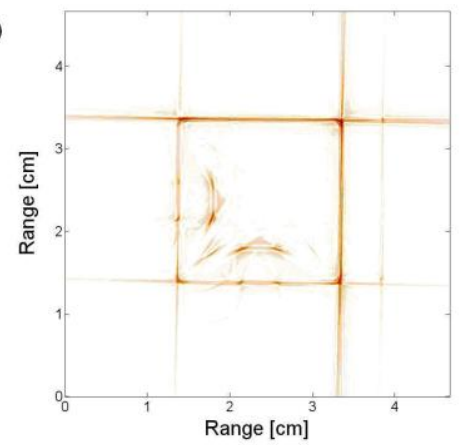

(b)

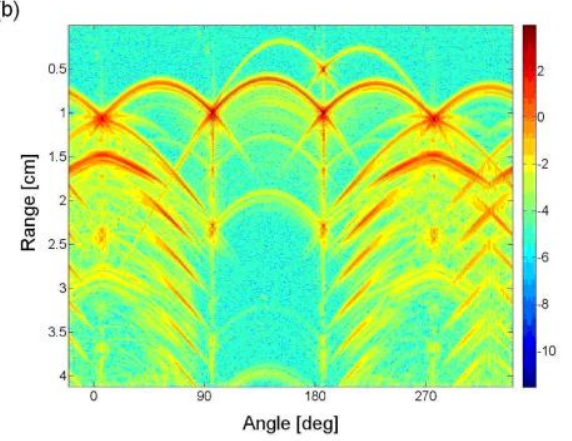

(d)

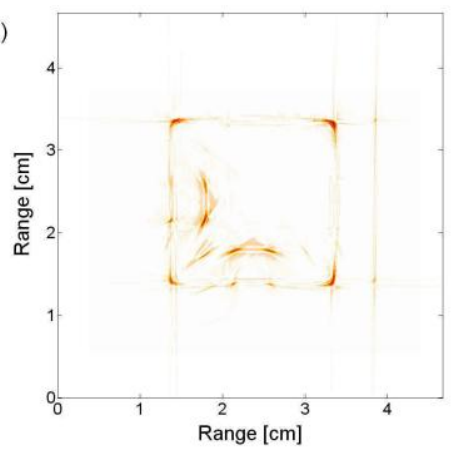

Fig. 6. (a) Metal test target (b) Logarithm of the instantaneous amplitude of THz waveforms scattered from the metal test target. (c) Cross section of the test target reconstructed using filtered back projection algorithm (d) Cross section of the test target reconstructed using filtered back projection algorithm with windowing out flat surface reflections.

Finally we have applied the FBP algorithm, with and without windowing on the data from the model aircraft sinogram in Fig. 4(c), and the results are shown in Fig. 7. The outline shape of the airplane can be easily recognized. Also particular scatterers such as fuselage, tail, wings, end of the wings and even missiles are distinguishable. 
(a)

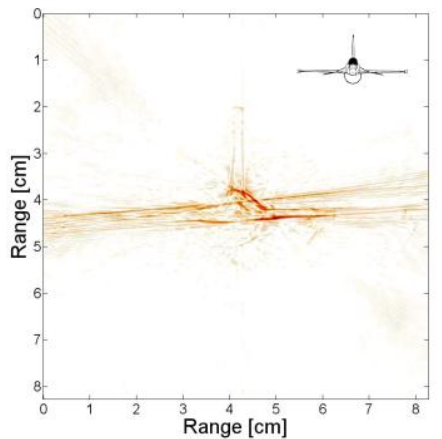

(b)

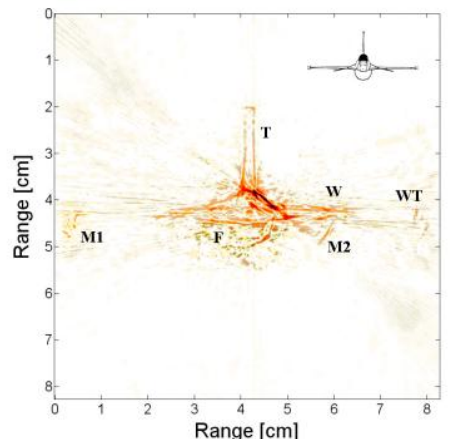

Fig. 7. Cross section of the scale model of the F-16 aircraft reconstructed using the filtered back projection algorithm on data from Fig. 4(c) without (a) and with (b) windowing out flat surface reflections. Letter marks indicate positions of different scattering parts of the airplane model: wing tips (WT), wing (W), tail (T), fuselage (F) and missiles (M1, M2).

\section{Conclusions}

We have presented a method for bistatic RCS measurements using THz waves. The calibrated setup has resolution of $0.2 \mathrm{~mm}$. We have performed a series of RCS measurements in the polar and azimuthal orientation on a scale model of the fighter aircraft F-16. By scaling wavelengths and models to real-scale platforms we can make detailed comparisons to $R C S$ of real sized aircrafts at standard $\mathrm{MHz} / \mathrm{GHz}$ frequencies. The application of a time domain system allows not only for determination of the total $R C S$ but it also provides $R C S$ values of particular elements of the model. Using the filtered back projection algorithm we are able to reconstruct a two-dimensional (and in principle also three-dimensional) visualization of all the scattering points. Our results show need for better algorithms, which could take into account the shape of the object and could compensate for diffraction, for improving the quality of reconstructed images.

\section{Acknowledgments}

We are grateful to the Danish Defense Acquisition and Logistics Organization and Photonics Academy Denmark for financial support and to Jørgen Dall from DTU Space and Otto S. Friis from DALO for informative discussions on radar techniques. 